\title{
Anestesia libre de opioides Revisión y reporte de un caso
}

Pérez-Atilano Tania Alexis*, Olvera-Romo Javier**

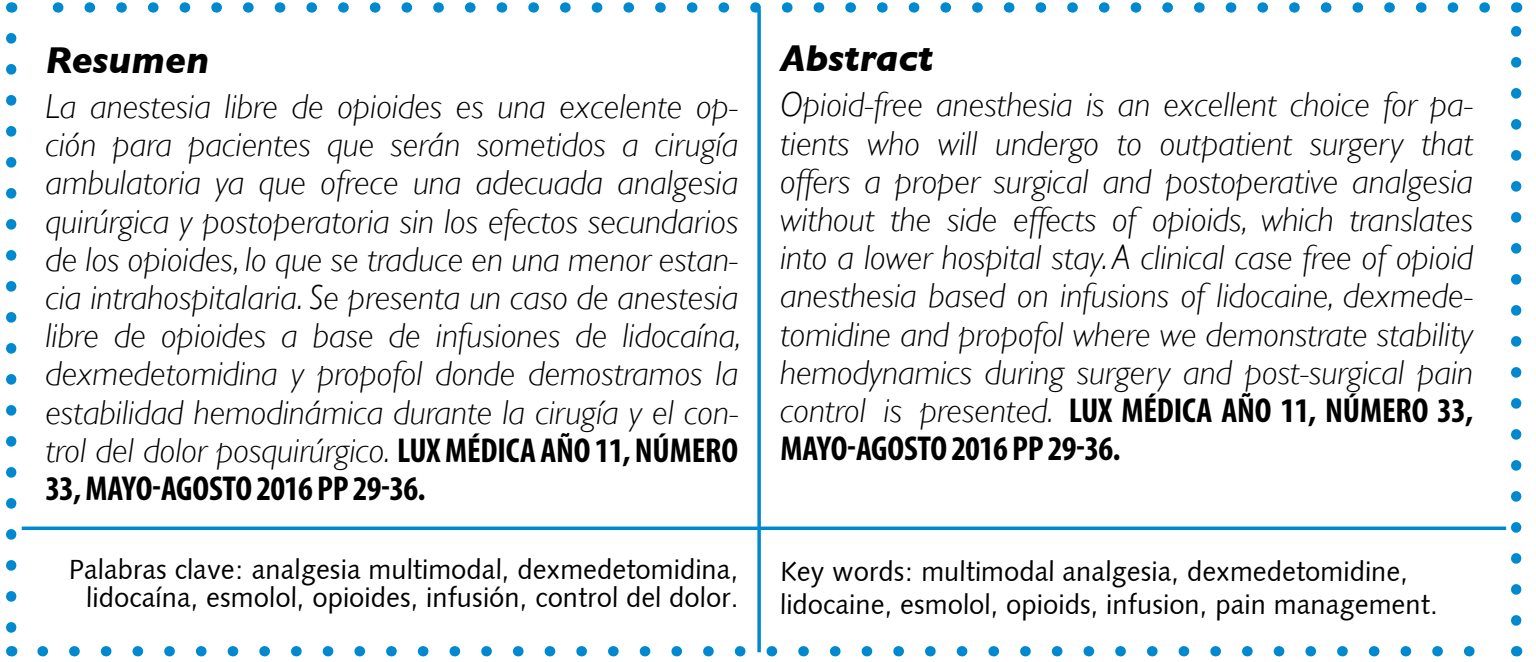

\section{Introducción}

La anestesia general es un método anestésico cuya base está dada por el cumplimiento de algunas características, tales como inconsciencia, amnesia, analgesia y parálisis, las cuales son necesarias para realizar la intubación traqueal con la menor repercusión simpática y lograr las condiciones óptimas para el procedimiento quirúrgico. Para su realización contamos con diversos fármacos, cada uno con propiedades distintas que bloquean la transmisión del dolor. Tras la primer anestesia dada por William Morton en 1846, se comenzaron a estudiar los efectos del éter en el sistema nervioso central (SNC) principalmente la inconsciencia, cualidad indispensable mas no única para lograr un adecuado plano anestésico; sin embargo, se presentaba inestabilidad hemodinámica y con ello múltiples

Médico Residente del primer año de Anestesiología del Centenario Hospital Miguel Hidalgo Médico Anestesiólogo Adscrito del Centenario Hospital Miguel Hidalgo

Correspondencia: Dr. Javier Olvera Romo. Centenario Hospital Miguel Hidalgo. Galeana Sur 465, Colonia Obraje. Código postal 20,000. Aguascalientes, Ags. México.Teléfono (01) 449994 6720.Correo electrónico: insanentwisted@hotmail.com 
complicaciones. En 1926 se inició con la práctica de "anestesias generales balanceadas" logrando mejores condiciones anestésicas. Hasta la década de los 60's se popularizó el uso de opioides entrando a la tendencia de anestesias basadas en la aplicación de grandes dosis de dichos fármacos logrando estados de analgesia exitosos pero con costos muy altos por múltiples y graves complicaciones. En 1993 se introdujo el concepto de "anestesia multimodal" la cual basa su técnica en la aplicación de diversos medicamentos que bloquean las diversas vías del dolor mediante la sinergia de sus efectos con la finalidad de ahorrar o incluso prescindir del uso de opioides. ${ }^{1-3}$

La tendencia actual es la realización, en la mayor medida de lo posible, de cirugías ambulatorias pues éstas representan mayor comodidad para el paciente y mayor beneficio económico para los hospitales debido a que resultan en menor ocupación de camas. ${ }^{2,4}$

|| || || |||||||||||||||||||||||||||||||||||||||||||||||||||||||||||||||||||||||||||||||||||||||||||||||||||||||||||||||||||

\section{Presentación del caso clínico}

Paciente femenino de 33 años de edad programada para realización de colecistectomía laparoscópica. Antecedentes de importancia: carga genética para diabetes mellitus tipo 2 (DM2), hipertensión arterial (HTA) y cáncer gástrico. Tabaquismo de 22 años de evolución con índice tabáquico de 5.5, alcoholismo intenso de 19 años de evolución suspendido siete meses previos a cirugía. Paciente roncadora crónica. Cesárea bajo anestesia regional sin complicaciones. Inicia su padecimiento actual seis meses previos a intervención con dolor abdominal de predominio en hipocondrio derecho asociado a ingesta de colecistoquinéticos, náusea y vómito, multitratada con inhibidores de la bomba de protones sin mejoría. Tras agudización acude a valoración donde con estudio de imagen se diagnostica colecistitis litiásica, se programa para realización de colecistectomía laparoscópica. En la valoración pre-anestésica se valora vía aérea con Mallampati clase II, Patil-Aldreti clase II, protrusión mandibular clase I, Bellhouse-Dore clase II, cuello grueso, resto de exploración sin datos relevantes. Peso $93 \mathrm{~kg}$, talla 1.60, índice de masa corporal (IMC) 36.6, presión arterial $110 / 60 \mathrm{mmHg}$, frecuencia cardiaca (FC) 60 latidos por minuto. Hemoglobina $(\mathrm{Hb})$ $14.7 \mathrm{~g} / \mathrm{dl}$, hematocrito 45.2, plaquetas 254,000; TP 14.4, TTP 28.8, INR 1.16. Firma consentimiento informado. Se le otorga una clasificación de ASA II, NYHA 1, riesgo tromboembólico bajo.

En quirófano se monitoriza a la paciente de forma no invasiva, se realizan mediciones de tensión arterial y frecuencia cardiaca para evaluar la respuesta dinámica durante los puntos críticos del evento quirúrgico-anestésico (cuadro 1). Se medica con midazolam $2 \mathrm{mg}$ IV y se inicia con inducción a base de bolo de dexmedetomidina 93mcg para pasar en 10min (dosis de $1 \mathrm{mcg} / \mathrm{Kg}$ ), durante la administración del bolo de impregnación se presenta episodio de bradicardia registrando FC de $45 \mathrm{lpm}$ por lo que se decide aplicación de atropina $1 \mathrm{mg}$ IV con el posterior mantenimiento de FC $>60$. Se comple- 
menta inducción con propofol bolo de 170mg IV, 140mg de lidocaína simple al 2\% en bolo intravenoso, se administra cisatracurio $10 \mathrm{mg}$ IV para facilitar la laringoscopia la cual se realiza de forma directa con hoja Macintosh 3, se observa Cormack Lehane clase III, se coloca cánula endotraqueal número 7.5 al primer intento, se corrobora su colocación mediante curva de capnografía y auscultación. Los parámetros de ventilador fueron Vt 500, FR 12, I:E 1:2, PEEP 4, con apoyo de oxígeno a 2L/min con FiO2 al 100\% manteniendo saturación de oxígeno del 100\%, se monitoriza ETCO2 el cual se mantiene durante la cirugía dentro del rango $29-34 \mathrm{mmHg}$. Mantenimiento anestésico: infusión de dexmedetomidina (la cual se inicia inmediatamente tras bolo de inducción) a dosis calculada de $0.5 \mathrm{mcg} / \mathrm{kg} / \mathrm{hr}$ quedando en infusión de $46.5 \mathrm{mcg} / \mathrm{kg} /$ hr, infusión de propofol (que se inicia tras terminar bolo de inducción) para mantener concentración plasmática $(\mathrm{Cp})$ de $2 \mathrm{mcg} / \mathrm{kg} / \mathrm{min}$, a velocidad de infusión de $33.4 \mathrm{ml} /$ hr, infusión de lidocaína simple al $2 \%$ (que se inicia tras terminar bolo de inducción) con dosis calculada de $2 \mathrm{mg} / \mathrm{kg} / \mathrm{hr}$ que en infusión representa 140mg/hr. Se solicita se infiltre piel a incidir con bupivacaína al $0.5 \%$. Medicamentos adyuvantes: metamizol 2 gr IV, dexametasona $8 \mathrm{mg}$ IV, metoclopramida $10 \mathrm{mg}$ IV, y 20 minutos previos al término de la cirugía se administran $30 \mathrm{mg}$ IV de ketamina, se observa automatismo ventilatorio. Al final del procedimiento, se cierran infusiones de medicamentes (duración de infusiones de $90 \mathrm{~min}$ ), se aspiran secreciones y bajo criterios de extubación se retira cánula endotraqueal sin complicaciones, tiempo de despertar estimado de $8 \mathrm{mi}-$ nutos (tras suspender infusiones). Se evalúa escala numérica del dolor inmediatamente tras extubación con calificación de cero. Se traslada paciente a unidad de cuidados postanestésicos sin complicaciones, se revalúa escala numérica del dolor refiriendo nuevamente cero (tabla 1).

La evaluación del dolor se llevó a cabo dentro de las $24 \mathrm{hr}$ de permanencia de la paciente en el hospital refiriendo una calificación de 2-3 a las 12 hr del postquirúrgico, dicho dolor se controla adecuadamente con aplicación de ketorolaco 30 mg IV con posterior calificación del dolor de cero.

\section{Tabla I}

Se muestra los signos vitales y la evaluación del dolor.

\begin{tabular}{|c|c|c|c|c|c|c|c|}
\hline Signos vitales & $\begin{array}{c}\text { Signos } \\
\text { basales }\end{array}$ & Inducción & $\begin{array}{c}1^{\circ} \text { minuto tras } \\
\text { inducción }\end{array}$ & $\begin{array}{l}\text { Laringos- } \\
\text { copía }\end{array}$ & $\begin{array}{l}\text { Estímulo } \\
\text { quirúrgico }\end{array}$ & $\begin{array}{l}\text { Neumoperi- } \\
\text { toneo }\end{array}$ & $\begin{array}{l}\text { Signos } \\
\text { finales }\end{array}$ \\
\hline Tensión arterial & $126 / 73$ & $135 / 83$ & $119 / 78$ & $126 / 85$ & $126 / 81$ & $123 / 85$ & $107 / 66$ \\
\hline Frecuencia cardiaca & 62 & 86 & 69 & 69 & 70 & 79 & 71 \\
\hline \multicolumn{8}{|c|}{ Evaluación del Dolor } \\
\hline Hora de evaluación & $\begin{array}{c}\text { Emersión } \\
\text { de la } \\
\text { anestesia }\end{array}$ & $\begin{array}{l}\text { Ingreso a } \\
\text { UCPA }\end{array}$ & $\begin{array}{l}1 \text { \%hora pos- } \\
\text { tquirúrgica }\end{array}$ & $\begin{array}{l}\text { Egreso de } \\
\text { UCPA } \\
2^{\circ} \text { hora } \\
\text { postquirúr- } \\
\text { gica }\end{array}$ & $\begin{array}{l}6^{\circ} \text { hora pos- } \\
\text { tquirúrgica }\end{array}$ & $\begin{array}{l}\text { 12hora pos- } \\
\text { tquirúrgica }\end{array}$ & $\begin{array}{c}24^{\circ} \text { hora } \\
\text { postqui- } \\
\text { rúrgica } \\
\text { Previa alta }\end{array}$ \\
\hline END & 0 & 0 & 0 & 0 & 1 & $2-3$ & 0 \\
\hline
\end{tabular}

*END: escala numérica de evaluación del dolor, UCPA: Unidad de cuidados post-anestésicos 


\section{Justificación}

El uso de opioides durante el trans y el postquirúrgico está asociado a múltiples efectos adversos, como al síndrome de náusea y vómito, sedación prolongada, íleo paralítico, retención urinaria e hiperalgesia. ${ }^{5} \mathrm{Di}$ chas complicaciones representan molestia para el paciente y entorpecimiento en la dinámica administrativa puesto que obligan a una mayor estadía hospitalaria con la utilización de mayores recursos humanos, médicos, así como insumos. ${ }^{1,4}$

El manejo del dolor quirúrgico representa un desafío para el anestesiólogo, pues diversas estructuras se lesionan durante el procedimiento quirúrgico ${ }^{6}$ y son potencialmente causantes de dolor agudo postoperatorio con la posibilidad de convertirse en dolor crónico. ${ }^{1,2}$ La combinación de anestésicos y analgésicos no opioides pueden evitar y tratar de una forma efectiva el dolor ocasionado durante un procedimiento quirúrgico, logrando la menor cantidad de complicaciones debidas a uso de opioides. ${ }^{5}$

La analgesia multimodal es una tendencia innovadora, segura y totalmente aplicable en pacientes sometidos a cirugías ambulatorias como la colecistectomías laparoscópicas. Durante esta cirugía se ocasionan lesiones a diversos niveles que se traducen a la aparición de dolor somático, visceral y neuropático aunado a la incomodidad que implica el neumoperitoneo con la aparición de dolor referido. Este tipo de cirugía es un desafío para el control del dolor, que fácilmente se puede prevenir y controlar bajo un esquema de analgesia multimodal con el cual cubrimos de forma satisfactoria el bloqueo del dolor en cualquiera de sus orígenes, logrando prevenir la aparición de dolor crónico. 7,8

La anestesia libre de opioides tiene diversas aplicaciones dando beneficios en pacientes obesos o con síndrome de apnea obstructiva, en pacientes asmáticos, hiperreactantes pulmonares o bien con insuficiencia respiratoria, en paciente alérgicos a opioides o con dependencia a los mismos, en pacientes con antecedentes de hiperalgesia o síndrome doloroso regional complejo, en cirugía oncológica y ante situaciones en las que se requiera movilización temprana tras la intervención quirúrgica. ${ }^{3}$

\section{AGENTES UTILIZADOS EN ANESTESIA LIBRE DE OPIOIDES}

\section{Propofol}

Fármaco perteneciente al grupo alquifenol, altamente liposoluble, contiene $1 \%$ de propofol, $10 \%$ de aceite de soja, 2.25 .5 de glicerol y $1.2 \%$ de lecitina de huevo. En un medicamente inductor ya que tiene propiedades sedantes e hipnóticas, amnésicas y antieméticas dosis dependientes. ${ }^{5}$ Su metabolismo es hepático mediante conjugación, tiene un efecto de primer paso el cual es pulmonar donde se elimina aproximadamente el $30 \%$ del fármaco aplicado en bolo, el resto es eliminado por vía renal. Su farmacocinética corresponde a un modelo tricompartamental con semivida de distribución inicial de 2-8 min, su efecto máximo se alcanza en $90-100$ segundos y una semivida de eliminación de 4-7 horas. Para que el paciente despierte se necesita que su concentración plasmática disminuya al menos un $50 \%$, es por ello que el despertar tras su aplicación en bolo, e incluso en infusión, sea rápido (5-8 minutos). ${ }^{5}$ Sus efectos tienen lugar a nivel de receptores GABA ya que su unión a la subunidad $\beta$ de GABA A ocasiona hipnosis, su efecto antiemético y sensación de bienestar está dado por el aumento de dopamina en el núcleo acumbens que ocurre tras su distribución a SNC.9,10 Entre otros efectos de este fármaco encontramos la producción de apnea tras 30 segundos de su aplicación, dicha apnea es dosis dependiente, disminuye en un $25-40 \%$ la tensión arterial sistólica y en un $15-30 \%$ las resistencias periféricas. ${ }^{9}$

Este medicamento ha demostrado ser 
efectivo en evitar los despertares transanestésicos (estado en el cual el paciente bajo anestesia general es consciente de sucesos ocurridos durante la cirugía y es capaz de recordarlos y describirlos al término de ésta) tras administración de un bolo de inducción calculado dentro del rango de $1-3 \mathrm{mg} / \mathrm{kg}$, existen diversos estudios que demuestran que el propofol, a dosis previamente mencionadas, es capaz de mantener valores de índice biespectral (BIS) 10 por debajo de 60 puntos, valor que asegura un adecuado estado de inconsciencia en los pacientes. El BIS es método matemático que estudia los trenes de ondas de las señales (potencia, frecuencia y fases de onda) de un electroencefalograma, se expresa en un valor numérico que nos refleja un estado de conciencia, tenemos pues que un valor de 100 nos habla de un paciente despierto que responde a la orden verbal, un valor de 80 nos indica que el paciente responde ante una voz fuerte o bien a estímulos físicos, 60 puntos es el valor aceptado para decir que un paciente no va tener despertares trans-anestésicos pues con este puntaje el paciente será incapaz de responder a estímulos tanto verbales como dolorosos. ${ }^{10,11}$

\section{Lidocaína}

De la familia de las aminoamidas, el fármaco más estudiado para la realización de anestesia libre de opioides es la lidocaína. Su mecanismo de acción es mediante la unión a canales de sodio y el aumento de la liberación de glutamato logrando la inhibición de vías de señalización intracelular que culmina en el bloqueo del impulso nervioso. Se le han atribuido propiedades analgésicas, antihiperalgésicas y antiinflamatorias. Tiene una latencia de 5-10 minutos proporcionando una anestesia rápida e intensa con una duración de hasta $60 \mathrm{~min}$, su metabolismo es hepático con una vida media de eliminación de 120 minutos, sigue un modelo farmacocinético bicompar- tamental con un importante primer paso pulmonar y eliminación renal.5,12

En diversos estudios se ha aplicado lidocaína en infusiones y se ha encontrado adecuado control del dolor a las 6 horas de la intervención quirúrgica además de una significativa reducción del dolor asociado al movimiento y una pronta recuperación del funcionamiento y motilidad intestinal indispensable para la recuperación del paciente ambulatorio. ${ }^{5}$ Se ha observado que la infusión de lidocaína contribuye al ahorro en el consumo de opioides además de disminuir la náusea y vómito posquirúrgico. $^{12}$

Las infusiones de lidocaína se pueden manejar dentro del parámetro de 1.5 a $3 \mathrm{mg} / \mathrm{kg} / \mathrm{hr}$ y han demostrado ser exitosas en el bloqueo de la transmisión del dolor y logrando rangos de concentraciones plasmáticas seguras. ${ }^{5,13}$ Se recomienda que la administración de este fármaco inicie entre los10 a 30 minutos previos a la incisión quirúrgica ${ }^{13}$ ya que mejora la calidad de la anestesia y analgesia en el perioperatorio. ${ }^{5}$ Algunos autores sugieren la culminación de dicha Infusión dentro de un rango que va de 1-24 horas tras retirar la vesícula. 5,13

\section{Dexmedetomidina}

La dexmedetomidina es un fármaco agonista $\alpha 2$ adrenérgico selectivo, de metabolismo hepático, tiene una vida media de eliminación de 2-3hr, sigue un modelo farmacocinético de tipo tricompartimental, con unión a proteínas en un $94 \%$ y una eliminación predominantemente renal. Actúa directamente en los receptores ubicados en el SNC y en la médula espinal, específicamente en el locus ceruleus y en las astas dorsales respectivamente. ${ }^{9,14,15}$ Una característica muy importante de este fármaco es que no modifica la dinámica ventilatoria así como los valores de $\mathrm{SatO}_{2}$ y $\mathrm{ETCO}_{2}{ }^{9,16}$ convirtiéndolo en una excelente opción para pacientes ambulatorios y obesos en quienes se anticipe una vía aérea difícil. 
La dexmedetomidina tiene propiedades sedantes, ansiolíticas, analgésicas, y un efecto muy importante sobre la respuesta simpática ante el estrés ya que disminuye la liberación de norepinefrina. ${ }^{15}$ Dentro de sus efectos adversos tenemos la aparición de bradicardia, la cual puede llegar a ser severa, sin embargo presenta una adecuada respuesta tras la aplicación de atropina, algunos autores recomiendan su aplicación ya sea profiláctica o bien terapéutica a dosis de $0.02 \mathrm{mcg} / \mathrm{Kg}$ IV o bien $1 \mathrm{mg}$ intramuscular 30 minutos previos al evento quirúrgico. ${ }^{7,9,15}$

Existen varios estudios en los que se han aplicado infusiones de dexmedetomidina para observar sus beneficios en el transoperatorio así como en el posquirúrgico. En dichos estudios se ha concluido que disminuye la estancia del paciente en la unidad de cuidados postanestésicos ya que disminuye la incidencia del síndrome de náusea y vómito (por su efecto en la disminución del tono simpático) ${ }^{14}$ así como las dosis necesarias de antieméticos para su control durante las primeras 24 horas del postquirúrgico. ${ }^{7}$ Entre otros efecto benéficos encontramos excelente analgesia postquirúrgica ya que el dolor se presen- ta de una forma más tardía y menos intensa además que permite su control con analgésicos tipo AINES a dosis bajas.7,15,16 La dexmedetomidina es una excelente opción para el manejo anestésico ambulatorio ya que su vida media de eliminación es de 2 horas y tiene la característica de que su efecto analgésico cubre más allá de las primeras 24 horas del posquirúrgico. ${ }^{14,15} \mathrm{Se}$ ha observado que disminuye la dosis necesaria de propofol para alcanzar un índice biespectral (BIS) de <60pts logrando así un adecuado plano anestésico previniendo con ello el despertar transanestésico, ${ }^{8}$ así como también disminuye la concentración alveolar mínima (CAM) de los gases anestésicos necesaria para mantener la anestesia. Se ha observado que la dexmedetomidina disminuye el tiempo necesario para la extubación, la apertura ocular así como la recuperación (definiendo el tiempo de recuperación como el tiempo que transcurre entre la suspensión de la infusión y la obtención de una calificación de 9-10 en la escala de Aldrete (figura 1), incluso se ha descrito como agente único en cirugías menores con excelentes resultados analgésicos. $^{9}$

\section{Dosis}

En Europa se han realizado diversos protocolos de aplicación de anestesia libre de opioides en pacientes sometidos a cirugía bariátrica laparoscópica con excelentes resultados. El protocolo más importante se realizó en el hospital Saint Jan Brugge en Brujas, en dicho protocolo se proponen las dosis de impregnación e infusión de dexmedetomidina, ketamina, lidocaína, esmolol, sulfato de magnesio, propofol y rocuronio. $^{3}$ (tabla 2 )

En el año 2014 en Turquía, Bakan y cols realizaron un estudio en el que compararon infusiones de propofol, dexmedetomidina y lidocaína versus infusiones de propofol, fentanil/remifentanil y lidocaína en colecistectomías laparoscópicas, ellos describieron otro plan de dosis (tabla 2) que resultó igualmente exitoso. ${ }^{17}$ Dichas dosis fueron utilizadas en el plan anestésico otorgado a nuestra paciente. 


\section{Tabla 2}

Dosis del protocolo de Saint Jan Brugge

\begin{tabular}{|c|c|c|c|c|c|c|c|}
\hline Fármaco & Lidocaína & Dexmedetomidina & Esmolol & Propofol & Ketamina & $\begin{array}{l}\text { Sulfato de } \\
\text { magnesio }\end{array}$ & Rocuronio \\
\hline $\begin{array}{l}\text { Impregnación } \\
\text { (bolo) }\end{array}$ & $1 \mathrm{mg} / \mathrm{kg}$ & $0.5-1 \mathrm{mcg} / \mathrm{kg} / 5-7 \mathrm{~min}$ & $0.5-1 \mathrm{mg} / \mathrm{kg} / 10 \mathrm{~min}$ & $2.5 \mathrm{mg} / \mathrm{kg}$ & $0.2-0.5 \mathrm{mg} / \mathrm{kg} / 5-7 \mathrm{~min}$ & $40 \mathrm{mg} / \mathrm{kg}$ & $0.6-1 \mathrm{mg} / \mathrm{kg}$ \\
\hline Infusión & $25 \mathrm{mcg} / \mathrm{kg} / \mathrm{min}$ & $0.5-1 \mathrm{mcg} / \mathrm{kg} / \mathrm{hr}$ & $5-15 \mathrm{mcg} / \mathrm{kg} / \mathrm{min}$ & $\begin{array}{l}120 \mathrm{mcg} / \mathrm{kg} / \mathrm{min} \\
{ }^{*} \text { gas anestésico }\end{array}$ & $0.125-0.3 \mathrm{mcg} / \mathrm{kg} / \mathrm{min}$ & $10 \mathrm{mcg} / \mathrm{kg} / \mathrm{hr}$ & $\begin{array}{c}\quad \begin{array}{c}1 \mathrm{mg} / \mathrm{kg} \\
/ \mathrm{hr} \text { según TOF }\end{array}\end{array}$ \\
\hline
\end{tabular}

Dosis utilizadas por Bakan y cols. en Turquía. Dosis utilizadas en nuestro caso

\begin{tabular}{|cccc|}
\hline Fármaco & Dexmedetomidina & Lidocaína & Propofol \\
\hline Impregnación (bolo) & $0.5-1 \mathrm{mcg} / \mathrm{kg} / 10 \mathrm{~min}$ & $1.5 \mathrm{mg} / \mathrm{kg}$ & $1.5-2.5 \mathrm{mg} / \mathrm{kg}$ \\
\hline Infusión & $0.3-0.5 \mathrm{mcg} / \mathrm{kg} / \mathrm{hr}$ & $1.5 \mathrm{mg} / \mathrm{kg} / \mathrm{hr}$ & $2-12 \mathrm{mg} / \mathrm{kg} / \mathrm{hr}$ \\
\hline
\end{tabular}

Figura 1. Escala de Recuperación de Aldrete.17

\begin{tabular}{llc} 
& Definición & Puntuación \\
\hline Actividad & Capaz de mover los cuatro miembros voluntariamente o bajo comando & 2 \\
\hline & Capaz de mover dos miembros voluntariamente o bajo comando & 1 \\
\hline & Incapaz de mover los miembros voluntariamente o bajo comando & 0 \\
\hline Respiración & Capaz de respirar profundamente o toser libremente & 2 \\
\hline & Disnea o respiración limitada & 1 \\
\hline & Apnea & 0 \\
\hline Circulación & Presión arterial $\pm 20 \%$ del nivel pre-anestésico & 2 \\
\hline & Presión arterial $\pm 20-50 \%$ del nivel pre-anestésico & 1 \\
\hline & Presión arterial $\pm 50 \%$ del nivel pre-anestésico & 0 \\
\hline Consciencia & Totalmente despierto & 2 \\
\hline & Despierto al llamado & 1 \\
\hline & No responde & 0 \\
\hline Saturacion de $\mathrm{O}_{2}$ & Mantiene $>92 \%$ en aire ambiente & 2 \\
\hline & Precisa inhalar $\mathrm{O}_{2}$ para mantener la saturación de $\mathrm{O}_{2}>90 \%$ & 1 \\
\hline & Saturación de $<90 \%$ incluso con suplementación de oxigeno & 0 \\
\hline
\end{tabular}

\section{Conclusiones}

El dolor quirúrgico, ya sea en su etapa transquirúrgica o postquirúrgica, es de origen multifactorial ya que es el resultado de lesiones cutáneas, viscerales y nerviosas, y, si no es tratado oportunamente y eficientemente, se traducirá en complicaciones como inestabilidad hemodinámica, despertar transanestésico y en dolor crónico. La anestesia libre de opioides se basa en la aplicación de analgesia multimodal para prevenir y tratar el dolor con la misma eficacia de la analgesia dada por derivados morfínicos pero sin los efectos secundarios de éstos. Para la aplicación de anestesia libre de opioides contamos con un gran arsenal de fármacos que han demostrado su eficacia al ser aplicados en bolos iniciales y continuados como infusiones. 
En nuestra experiencia con la aplicación de anestesia libre de opioides observamos un claro éxito en el control de la estabilidad hemodinámica, por lo que nos hace pensar que alcanzamos un adecuado plano anestésico (el cual no tuvimos forma objetiva de medir), un adecuado control del dolor transquirúrgico (evaluando la estabilidad hemodinámica mediante la medición de cifras de tensión arterial y frecuencia cardiaca) y dolor post-operatorio (mediante la evaluación periódica de la escala numérica del dolor la cual fue en el rango 2-3 con adecuado control tras administración de AINES).

Podemos concluir que esta técnica anestésica es prometedora y aplicable a nuestro medio, que representa una excelente opción para pacientes ambulatorios o que requieran movilización temprana. Sus limitantes son la aparición de bradicardia que remite tras aplicación de atropina y la sedación con duración menor a $2 \mathrm{hr}$ tras suspensión de infusiones. Entre sus beneficios tenemos incidencia de náusea y vómito baja, presenta un retardo en la aparición del dolor así como la disminución de su intensidad y facilita su tratamiento ambulatorio con fármacos de uso habitual y seguro como lo son los AINES.

\section{Bibliografía}

1. Elvir-Lazo, Ofelia y White, Paul. The role of multimodal analgesia in pain management after ambulatory surgery. Curr Opin Anaesthesiol. 2010; 23(6):697-703. doi: 10.1097/ACO.0b013e32833fad0a.

2. White $P$, Henrik K. improving postoperative pain management. Anesthesiology,2010;112:220-225.

3. Vargas-Hernández J. Anestesia libre de opiodes. Revista Mexicana de Anestesiología, 2014;37(1): S24-S27.

4. Coloma M, Chiu JW, White PF, Armbruster SC. The use of esmolol as an alternative to remifentanil during desflurane anesthesia for fast-track outpatient gynecologic laparoscopic surgery. Anesth Analg. 2001;92(2):352-7.

5. Mefkur B, Tarik U, Ufuk T, Harun U, Mehmet B, Huseyin K, Ziya S. Anestesia venosa total libre de opiáceos, con infusiones de propofol, dexmedetomidina y lidocaína para la colecistectomía laparoscópica: estudio prospectivo, aleatorizado y doble ciego. Rev Bras Anestesiol 2015;65(3):191-199.

6. Collard V, Mistraletti G, Taqi A, Asenjo JF, Feldman LS, Fried GM, Carli F. Intraoperative esmolol infusion in the absence of opioids spares postoperative fentanyl in patients undergoing ambulatory laparoscopic cholecystectomy. Anesth Analg. 2007 ;105(5):1255-1262.

7. Blaudszun $G$, Lysakowski $C$, Elia $N$, Tramèr MR. Effect of perioperative systemic ?2 agonists on postoperative morphine consumption and pain intensity: systematic review and meta-analysis of randomized controlled trials. Anesthesiology. 2012;116(6):1312-22. doi:10.1097/ALN.0b013e31825681cb.

8. Park JK, Cheong SH, Lee KM, Lim SH, Lee JH, Cho K, Kim MH, Kim HT. Does dexmedetomidine reduce postoperative pain after laparoscopic cholecystectomy with multimodal analgesia? Korean J Anesthesiol. 2012 Nov;63(5):436-40. doi: 10.4097/kjae.2012.63.5.436.

9. Raouf A, El Ghoneimy M. Dexmedetomidine versus fentanyl as adjuvant to propofol: comparative study in children undergoing extracorporeal shock wave lithotripsy. Eur J Anesthesiol. 2010; 27:1058-1064.

10. Tardío-Flores Rodrigo A., Sejas-Clavijó Jacquie, Castellón-Sejas Virginia, Bustamante Carmen, Orozco-
Cadima Anell. Utilidad del índice Biespectral en la Monitorización de la Conciencia Durante la Anestesia General. Rev Cient Cienc Méd [Internet]. 2010 Dic [citado 2016 Jun 27] ; 13( 2 ): 69-72. Disponible en: http://www.scielo.org.bo/scielo.php?script=sci arttext\&pid=S1817-74332010000200004\&lng=es.

11. Higuera Medina L. ¿Es recomendable el uso del índice biespectral en todo paciente bajo anestesia? Revista Mexicana de Anestesiología, 2010; 33(1):S64-S66.

12. Vigneault $L$, Turgeon AF, Côté $D$, Lauzier $F$, Zarychanski R, Moore L, McIntyre LA, Nicole PC, Fergusson DA. Perioperative intravenous lidocaine infusion for postoperative pain control: a meta-analysis of randomized controlled trials. Can J Anaesth. 2011;58(1):22-37. doi: 10.1007/s12630-010-9407-0.

13. Wu CT, Borel CO, Lee MS, Yu JC, Liou HS, Yi HD, Yang CP. The interaction effect of perioperative cotreatment with dextromethorphan and intravenous lidocaine on pain relief and recovery of bowel function after laparoscopic cholecystectomy. Anesth Analg. 2005; 100(2):448-53.

14. Tufanogullari $B$, White $P F$, Peixoto $M P$, Kianpour $D$, Lacour T, Griffin J, Skrivanek G, Macaluso A, Shah M, Provost DA. Dexmedetomidine infusion during laparoscopic bariatric surgery: the effect on recovery outcome variables. Anesth Analg. 2008 ;106(6):1741-8. doi: 10.1213/ane.0b013e318172c47c.

15. Arain SR, Ruehlow RM, Uhrich TD, Ebert TJ. The efficacy of dexmedetomidine versus morphine for postoperative analgesia after major inpatient surgery. Anesth Analg. 2004;98(1):153-158.

16. Gurbet Alp, Basagan-Mogol E, Gurkan T, Ugun F, Kaya $\mathrm{N}$, Ozcan B. Intraoperative infusion of dexmedetomidine reduces perioperative analgesic requirements. Can J Anesth 2006;53(7):646-652.

17. Türk Hacer ?ebnem, Aydo?mu? Meltem, Ünsal Oya, Köksal Hakan Mustafa, Açik Mehmet Eren, Oba Sibel. Sedación y analgesia en colonoscopia electiva: propofol-fentanilo versus propofol-alfentanilo. Rev. Bras. Anestesiol. 2013;63(4):353-358. doi.org/10.1016/j. bjan.2012.07.006. 